\title{
Prognostic Impact of Flow Cytometric Measurement of G Protein-Coupled Receptor 56 (GPR56) in Acute Myeloid Leukemia
}

\author{
Hanaa M. Sayed Afifi( ${ }^{(1)}$, Mai A. Rashad (1), Haitham M. Abdelbary ${ }^{(2)}$, Rasha A. El-Gamal ${ }^{(1)}$ \\ Departments of ${ }^{(1)}$ Clinical Pathology and ${ }^{(2)}$ Internal Medicine and Bone Marrow Transplantation, \\ Faculty of Medicine, Ain Shams University, Egypt \\ Corresponding author: Rasha A. El-Gamal; Mobile: 01145405005; Email: rashaelgamal@med.asu.edu.eg
}

\begin{abstract}
Background: A variety of factors influence the prognosis of acute myeloid leukemia (AML), and leukemic stem cells (LSCs) are one key prognostic factor that predicts poor prognosis over the course of the disease. G protein-coupled receptor 56 (GPR56) was presented as a new human LSC marker in AML patients. The engraftment potential of GPR56+ cells selected from AML specimens helps the spread of leukemia in mice, which supports the concept of using it as an LSC marker. Aim of the Work: The objective of this study was to use flow cytometry to investigate GPR56 expression in newly diagnosed AML patients. The results of GPR56 expression were linked to the patients' clinical outcomes. Subjects and methods: Forty AML patients and 20 healthy control subjects were evaluated for GPR56 expression on myeloblasts. At the end of induction treatment, bone marrow aspirates were obtained for assessment of morphological remission and minimal residual disease analysis. Results: GPR56 levels were significantly higher in control subjects than in AML patients. AML patients who failed to achieve complete remission were more likely to show high levels of GPR56. However, a relationship to long term follow up data was not evident in our results. Conclusion: Despite the lack of a direct prognostic influence of GPR56 on patient outcome or a relationship to prognostic cytogenetic subgroups, the association of GPR56 with a poor prognostic marker, early response to therapy, can be regarded an evidence of the speculated bad prognostic impact of GPR56.
\end{abstract}

Keywords: Acute myeloid leukemia; Complete remission; Flow cytometry; G protein-coupled receptor 56; prognosis.

\section{INTRODUCTION}

Acute myeloid leukemia (AML) is a diverse disease that is distinguished by unregulated proliferation of leukemic myeloblasts, which interferes with the formation of normal blood cells. AML is the most prevalent acute leukemia in adults, and its incidence continues to increase with age ${ }^{(1)}$. Relapse remains a common sequel in AML, as the 5-year survival of $30-45 \%$ has been reported, which differs according to age. Recognition of AML prognostic factors have become increasingly important for the choice of the appropriate therapy ${ }^{(2,3)}$.

A variety of factors influence the prognosis of AML, and leukemic stem cells (LSCs) are one key prognostic determinant that can predict disease relapse ${ }^{(4,5)}$. LSCs have been shown to be located in the CD34+ CD38- compartment ${ }^{(6)}$, and various markers have been described to identify LSCs particularly anti-CD44, anti-CD33, and anti-CD123 ${ }^{(7)}$.

The surface $G$ protein-coupled receptor 56 (GPR56) was presented as a new human LSC marker in AML patients. GPR56 is one of the adhesion family members that belongs to the $G$ protein-coupled receptors (GPCR) superfamily and has been connected to human brain developmental abnormalities (8). Overexpression of GPR56 in cancer cells has been shown to inhibit tumour development and metastasis in melanoma cell lines, while GPR56 is involved in tumour cell adhesion in glioma cells ${ }^{(9)}$. Although the role of GPR56 in hematopoietic stem cells (HSCs) is uncertain, it was discovered in a family of LSC-related genes that are linked to a dismal AML prognosis.
Adherence, migration, and mobilization of HSC in BM niches are influenced by the small GTPases, while GPR56 modulates RhoA signaling implicated in cellular adhesion ${ }^{(9)}$.

In a study by Pabst and coworkers, GPR56+ cells were found to have an engraftment capability in both the CD34+ and CD34- fractions sorted from specific AML samples, and they played an important role in leukemia growth in mice. Hence, the authors have proposed GPR56 to define a new LSC compartment that is distinct from the CD34+CD38- LSC phenotype ${ }^{(10)}$.

Stemming from this speculated prospect of GPR56 in AML, the objective of this study was to use flow cytometry to investigate GPR56 expression in newly diagnosed AML patients. The results of GPR56 expression are to be linked to the patients' clinical outcomes as well as other relevant laboratory data.

\section{PATIENTS AND METHODS}

This study involved 40 patients with newly diagnosed AML, all of them were evaluated and treated at Hematology-Oncology Department, Ain Shams University Hospitals, during the period from November 2016 to July 2017. In addition, 20 nonleukemic patients in whom bone marrow (BM) aspirates were obtained as a part of their diagnostic work up were included as healthy control group.

\section{Ethical approval}

All patients and controls gave verbal informed consents. The work was approved by Ain Shams University's Local Research Ethical 


\section{Committee and it was done in accordance with the Helsinki Declaration.}

AML diagnosis and subcategorization were established fulfilling the World Health Organization criteria (revised version, 2017) ${ }^{(11)}$. Patients received treatment according to the National Comprehensive Cancer Network guidelines (12). Patients were evaluated at day 28 of starting induction therapy by assessment of complete remission (CR) status and then followed up for the disease course for periods ranging from 2 to 20 months. All of the patients were given a comprehensive medical history and clinical assessment. Peripheral blood (PB) samples were collected from all enrolled subjects and tested for complete blood count (CBC) (Coulter LH 750; Beckman Coulter Inc., Fullerton, California, USA). BM aspirate samples were collected and used to make BM smears (Romanowsky stained) and were assessed, along with PB smears, to determine the blast count, report dysplastic morphology, and define its FAB (French-American-British) subtype (with the help of cytochemical stains).

Flow cytometric analysis was performed using EDTA-anticoagulated BM aspirate or PB specimens and were processed within 24 hours after collection. Samples were incubated with monoclonal antibodies or its isotypic control for 20 minutes at room temperature protected from light. Ammonium chloride-based erythrocyte lysing solution was added $[8.29 \mathrm{~g}(0.15 \mathrm{M})$ $\mathrm{NH}_{4} \mathrm{Cl}, 1 \mathrm{~g}(10 \mathrm{mM}) \mathrm{KHCO}_{3}, 0.037 \mathrm{~g}(0.1 \mathrm{mM})$ EDTA, and $1 \mathrm{~L}$ distilled water, adjusted to $\mathrm{pH} 7.3$ ] for 10 minutes followed by a standard wash step using phosphate buffered saline. Samples were acquired on Coulter Navios 6-color flow cytometer (Beckman Coulter, Miami, FL, USA). The antibody combinations used were CD13-PE, CD33-PE CD14-FITC, CD64-FITC, CD117PE, CD34-FITC, myeloperoxidase-FITC and CD45-PC5 (all monoclonal antibodies were obtained from Beckman Coulter reagents, Brea, CA, USA). PE-conjugated monoclonal anti-human anti-GPR-56 reagent (Miltenyl Biotec GmbH, Bergisch Gladbach, Germany) was used to test for its expression on myeloblasts. At least 10,000 events were considered sufficient for reasonable display of phenotypes. Identification of myeloblasts was based mainly on CD45dim/side scatter characteristics and they were confirmed afterwards by CD34 and/or CD117 expression. Monocytic leukemia was characterized by the pattern of CD64/CD14 expression. Samples were considered positive for a certain marker when the marker was expressed by at least $20 \%$ of cells, except for CD34 and myeloperoxidase where their expression by only $10 \%$ of cells was enough to indicate positivity (13). GPR56 expression was assessed on blasts and blast equivalents (if present) for each patient, and the percentage of blasts positive for GPR56 was calculated. In addition, the mean fluorescence intensity (MFI) values of GPR56 expression were determined and a negative control was used to compute the MFI ratio.

Conventional cytogenetic analysis and/or molecular cytogenetics using fluorescence in situ hybridization (FISH) probes was done for AML patients, according to which they were divided into three prognostic cytogenetics categories: favorable, intermediate, or adverse prognostic cytogenetics categories, based on the revised UK Medical Research Council criteria for AML ${ }^{(14)}$, and revised 2017 WHO criteria for diagnosis of AML ${ }^{(11)}$. Patients who were allocated in the favorable prognostic group were tested for FLT-3 and NPM1 mutations for final confirmation of the good prognostic state.

\section{Statistical analysis}

Data were analyzed using IBM@ SPSS $\odot$ Statistics, version 22 (IBM@ Corp., Armonk, NY, USA). The mean, standard deviation (SD), median and interquartile range (IQR) were used to describe the data. The statistical significance of the difference between two GPR56-based study group medians was determined using the Mann Whitney $U$ test. Categorical variables were presented as number (\%) and differences were compared using Fisher's exact test. To compare more than 2 medians, Kruskal-Wallis test was utilized. Receiver-operating characteristic (ROC) curve analysis was employed to assess the value of GPR56 in distinguishing patients who achieved complete remission (CR) at day 28. The DeLong method was used to compare the areas under individual ROC curves. Kaplan Meier curves were performed for examining overall survival and event free survival followed by Log Rank test to assess statistically significant change between groups. Statistical significance was defined as a $P$ value of less than 0.05 .

\section{RESULTS}

Descriptive and demographic data of AML patients

The study included 40 newly diagnosed AML patients, 22 males (55\%) and 18 females (45\%) with $\mathrm{M}$ : $\mathrm{F}$ ratio 1.2:1; their ages ranged from $18-73$ years with mean value of $41.75 \pm 15.54$. The control subjects comprised 13 males $(65 \%)$ and 7 females $(35 \%)$ with $\mathrm{M}$ : $\mathrm{F}$ ratio 1.8:1; their ages ranged from 18-70 years with mean value of $40.45 \pm 17.27$ years. The mean BM blast count at presentation was $66.35 \%+17.28$ (range: 20\%-90\%). Using immunophenotyping data together with the morphology, 27 patients $(67.5 \%)$ were diagnosed as M1/M2, 8 patients (20\%) were diagnosed as M3, and 5 patients $(12.5 \%)$ were diagnosed as M4/M5. Conventional cytogenetic and FISH analysis were performed where $28(70 \%)$ patients had normal karyotype, 3 patients $(7.5 \%)$ had $\mathrm{t}(8 ; 21)(\mathrm{q} 22 ; \mathrm{q} 22), 8$ patients $(20 \%)$ had $\mathrm{t}(15 ; 17)(\mathrm{q} 22 ; \mathrm{q} 21)$, and 1 patient $(2.5 \%)$ had trisomy 21 . Accordingly, together with the available results of FLT3 and NPM1 testing, 10 patients $(25 \%)$ were found to have favorable prognostic cytogenetics, 29 patients $(72.5 \%)$ with intermediate prognostic cytogenetics, and 1 patient $(2.5 \%)$ had adverse prognostic cytogenetics.

In the present study, the remission status was described following the European LeukemiaNet (ELN) nomenclature ${ }^{(15)}$. Patients meeting the criteria 
of complete remission (CR), CR with incomplete hematologic recovery (CRi) and $\mathrm{CR}$ without minimal residual disease $\left(\mathrm{CR}_{\mathrm{MRD}}\right)$ ) were included in the group of CR; otherwise, treatment response was described as partial remission (PR). Accordingly, 21 patients (52.5\%) achieved CR and 19 patients (47.5\%) showed
PR. Moreover, follow-up of the patients has revealed that 15 patients maintained the CR state, 4 patients relapsed, and 13 died. Thus, outcome was classified as good outcome (patients who maintained CR) and poor outcome (relapse and death cases). Table 1 shows the clinical and laboratory data of AML patients.

Table 1: Laboratory and clinical data of AML patients

\begin{tabular}{|c|c|c|}
\hline Parameter & & Mean \pm SD (range) or $\mathrm{n}(\%)$ \\
\hline TLC & $\times 10^{9} / \mathrm{L}$ & $49.19 \pm 6.91$ \\
\hline$\overline{\mathrm{Hb}}$ & $\mathrm{g} / \mathrm{dL}$ & $7.69 \pm 1.72$ \\
\hline Platelets & $\times 10^{9} / \mathrm{L}$ & $59.1 \pm 6.5$ \\
\hline PB blasts & $\%$ & $57.1 \pm 9$ \\
\hline BM blasts & $\%$ & $66.4 \pm 7.3$ \\
\hline \multirow{3}{*}{ AML FAB subtype } & $\mathrm{M} 1 / \mathrm{M} 2$ & $27(67.5)$ \\
\hline & M3 & $8(20)$ \\
\hline & M4/ M5 & $5(12.5)$ \\
\hline \multirow{3}{*}{ Cytogenetics } & Favorable & $10(25)$ \\
\hline & Intermediate & $29(72.5)$ \\
\hline & Unfavorable & $1(2.5)$ \\
\hline \multirow{2}{*}{$\begin{array}{l}\text { Response to treatment at } \\
\text { D28 }\end{array}$} & $\mathrm{CR}$ & $21(52.5)$ \\
\hline & $\overline{P R}$ & $19(47.5)$ \\
\hline \multirow{3}{*}{ Outcome } & Good Maintenance of CR & $15(46.9)$ \\
\hline & \multirow{2}{*}{\begin{tabular}{|ll} 
Bad & $\begin{array}{l}\text { Relapsed } \\
\text { Died }\end{array}$ \\
\end{tabular}} & $4(12.5)$ \\
\hline & & $13(40.6)$ \\
\hline
\end{tabular}

AML: acute myeloid leukemia; BM: bone marrow; CR: complete remission; FAB: French-American-British; Hb: hemoglobin; PR: partial remission; TLC: total leucocytic count; PB: peripheral blood.

\section{Relationship between FAB classification or cytogenetics and response to therapy and patient outcome}

A statistically significant relationship was found between immunophenotyping classification and achievement of CR at day 28 where PR was more prevalent among M4/M5 cases. On the other hand, there was no statistically significant relationship between hematological remission at day 28 and cytogenetics. As regards patients' outcome, there was no significant differences in the prevalence of AML-FAB subtypes or cytogenetics study results between patients with good outcome and those with poor outcome (Table 2).

Table 2: Relation between FAB classification and cytogenetics with outcome of patients

\begin{tabular}{|c|c|c|c|c|c|c|c|}
\hline \multirow{2}{*}{\multicolumn{2}{|c|}{ Prognostic measure }} & \multicolumn{3}{|c|}{ AML FAB subtype } & \multicolumn{3}{|c|}{ Cytogenetics-based prognostic category } \\
\hline & & $\mathrm{M} 1 / \mathrm{M} 2$ & M3 & M4 / M5 & Favorable & Intermediate & Unfavorable \\
\hline \multirow{3}{*}{$\begin{array}{l}\text { Response to } \\
\text { treatment at } \\
\text { D28 }\end{array}$} & CR & $17(63)$ & $4(50)$ & $0(0)$ & $7(70)$ & $14(48.3)$ & $0(0)$ \\
\hline & PR & $10(37)$ & $4(50)$ & $5(100)$ & $3(30)$ & $15(51.7)$ & $1(100)$ \\
\hline & $P$ value & & 0.033 & & \multicolumn{3}{|c|}{0.281} \\
\hline Good outcome & $\begin{array}{r}\text { Maintenance of } \\
\text { CR }\end{array}$ & $11(55)$ & $4(50)$ & $0(0)$ & $3(50)$ & $12(48)$ & $0(0)$ \\
\hline \multicolumn{8}{|l|}{ Poor outcome } \\
\hline & Relapse & $3(15)$ & $0(0)$ & $1(25)$ & $1(16.7)$ & $3(12)$ & $0(0)$ \\
\hline & Death & $6(30)$ & $4(50)$ & $3(75)$ & $2(33.3)$ & $10(40)$ & $1(100)$ \\
\hline & $P$ value & \multicolumn{3}{|c|}{0.17} & \multicolumn{3}{|c|}{0.8} \\
\hline
\end{tabular}

AML: acute myeloid leukemia; CR: complete remission; D28: day 28; FAB: French-American-British; PR: partial remission. GPR56\% expression and MFI values in the study subjects, and in relation to studied variables

There was a statistically significant difference in GPR56\% expression and MFI between AML patients (76.9\% (IQR 56-94.5); 2.6 (IQR 2.15-4), respectively) and control subjects (96\% (IQR 93.3-98.4); 3.6 (IQR 3-5.1), respectively), where both parameters showed significantly higher values in control subjects than AML patients ( $\mathrm{P}$ value $=0.001$ and 0.004 , respectively).

GPR56\% expression and MFI values were studied in relation to other prognostic variables. Both parameters were significantly related to response to treatment at day 28, where higher GPR56\% expression and MFI values were associated with PR. On the other hand, both parameters were not associated with good or poor outcome of the disease, nor with immunophenotyping or cytogenetic analysis results (Table 3 ). 
Table 3: GPR56 \% expression and MFI values according to different variables

\begin{tabular}{|c|c|c|c|c|c|}
\hline \multirow{2}{*}{ Parameter } & & \multicolumn{2}{|l|}{ GPR56\% } & \multicolumn{2}{|l|}{ MFI } \\
\hline & & Median (IQR) & $P$ & Median (IQR) & $P$ \\
\hline \multirow{2}{*}{ Sex } & Male & $71(55.6-91.5)$ & \multirow{2}{*}{0.347} & $2.43(2.13-3.6)$ & \multirow{2}{*}{0.901} \\
\hline & Female & $90.1(52.05-98.05)$ & & $3(2.19-3.81)$ & \\
\hline \multirow{3}{*}{$\begin{array}{l}\text { AML FAB } \\
\text { subtypes }\end{array}$} & M1/M2 & $74.7(45.9-96.1)$ & \multirow{3}{*}{0.97} & $2.7(2.16-4)$ & \multirow{3}{*}{0.344} \\
\hline & M3 & $78(67.2-92.6)$ & & $3.1(2.22-3.9)$ & \\
\hline & M4/M5 & $88.2(28.7-98.3)$ & & $2.4(1.03-2.9)$ & \\
\hline \multirow{2}{*}{ Cytogenetics } & Favorable & $91.5(42-98.8)$ & \multirow{2}{*}{0.44} & $3(2.03-3.62)$ & \multirow{2}{*}{0.81} \\
\hline & Intermediate & $74.7(56.4-92.7)$ & & $2.69(2.14-3.9)$ & \\
\hline \multirow{2}{*}{$\begin{array}{l}\text { Response to } \\
\text { treatment at D28 }\end{array}$} & $\mathrm{CR}$ & $66.3(33.7-93.2)$ & \multirow{2}{*}{0.001} & $2.19(1.53-2.88)$ & \multirow{2}{*}{0.011} \\
\hline & PR & $97.5(86.6-99.3)$ & & $3(2.4-4.16)$ & \\
\hline Good Outcome & Maintenance of CR & $68(48-90.7)$ & \multirow{3}{*}{0.128} & $1.9(1.71-2.4)$ & \multirow{3}{*}{0.186} \\
\hline \multirow[t]{2}{*}{ Poor Outcome } & Relapse & $79(45.9-91.5)$ & & $2.4(2.1-4)$ & \\
\hline & Death & $93.3(61.5-98.8)$ & & $2.95(2.2-4)$ & \\
\hline
\end{tabular}

Abbreviations: AML: acute myeloid leukemia; CR: complete remission; D28: day 28; FAB: French-American-British; GPR: G protein-coupled receptor; PR: partial remission

Since no clear cutoff could be established for GPR56 positive expression in our AML patients (as control subjects showed higher GPR56 expression), we used the median values of GPR56\% and GPR56 MFI, 76.9\% and 2.6, to represent a cutoff value to categorize AML patients into high- and low- GPR56\% and GPR56 MFI expression groups, respectively. Those values were used to characterize patients and carry out survival analyses. The definitions stated by the International Working Group for Therapeutic Studies in AML to measure survival were implemented in our study (16). By the end of follow up period (2-20 months), the studied patients had mean EFS of 10.56 months (95\% CI: 8 13.12), and mean OS of 8.62 months (95\% CI: 6.64 - 10.61). Using the data of 32 patients of our cohort, the EFS and OS times were used to construct survival plots (Kaplan-Meier curves). The log rank analysis showed no statistically significant association between GPR56\% expression or MFI and either EFS or OS (Figure 1).

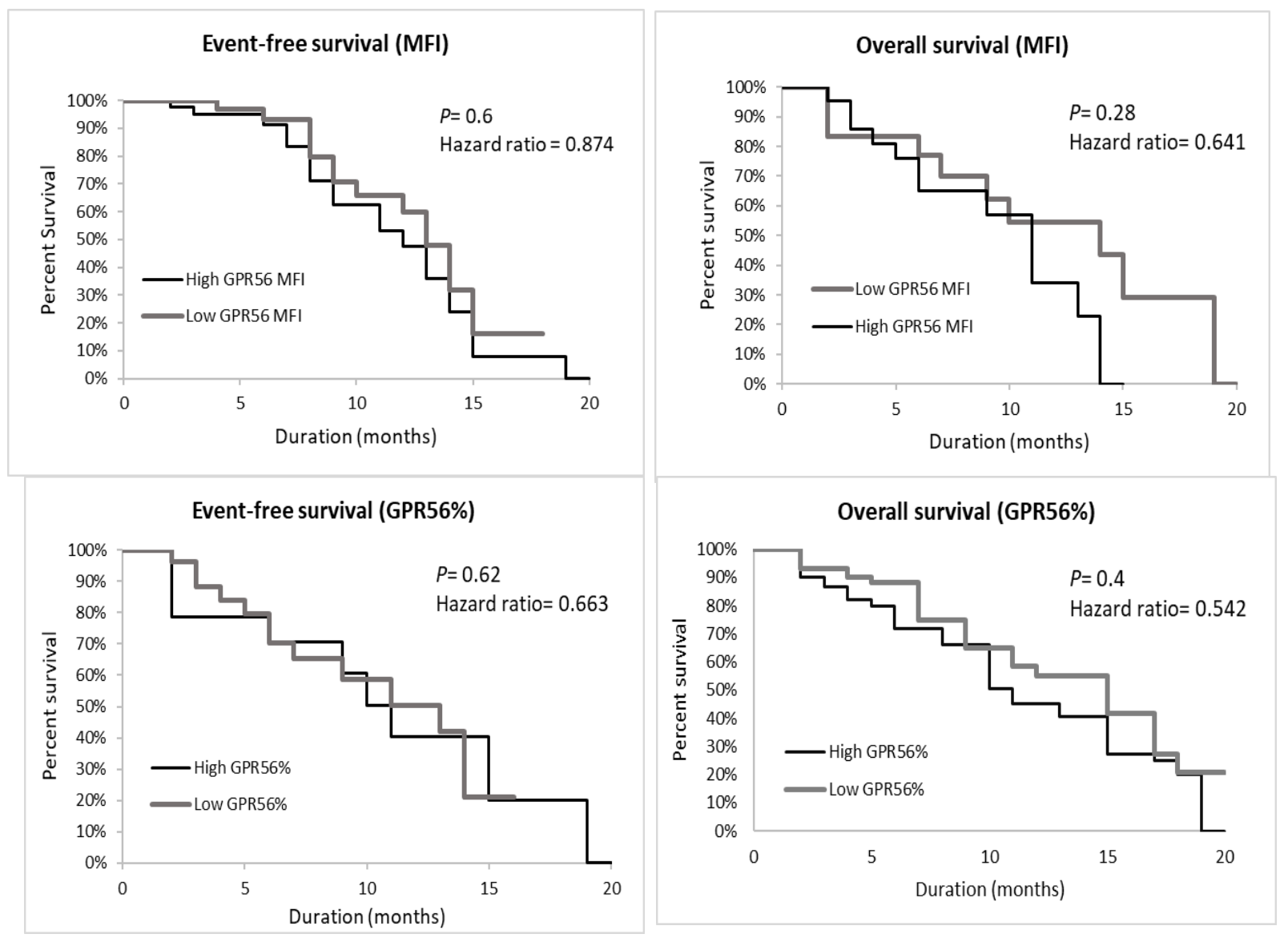

Figure 1. Kaplan-Meier curve for estimated EFS and OS in AML patients. 
Determination of a cutoff value for GPR56\% to predict response to induction treatment

ROC curve analysis was used to evaluate the ability of GPR $56 \%$ expression to predict failure to attain CR in AML patients, producing an area under the curve (AUC) of 0.781 (Figure 2). A cutoff point more than $91.2 \%$ was found useful to indicate incomplete remission with sensitivity $71.43 \%$, specificity $61.11 \%$, positive predictive value (PPV) $58.8 \%$ and negative predictive value (NPV) $73.3 \%$. Worth mentioning, ROC curve constructed to assess the ability of GPR56 MFI values to indicate the state of PR has revealed a low AUC $(0.546$; 95\% CI: 0.3620.73 ), negating its use to predict response to therapy.

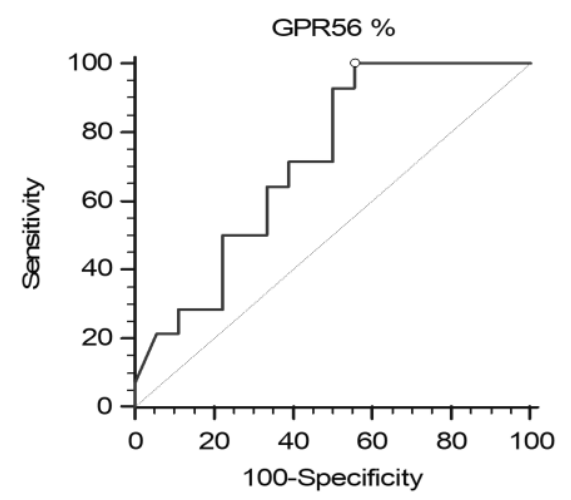

Figure 2. ROC curve of GPR56\% values in relation to remission status.

\section{DISCUSSION}

AML is described as having a characteristic hierarchical nature that emerges from a specific fraction of LSCs. Similar to HSCs, the normal counterpart, LSCs have the ability to self-renew and are responsible for the continuing growth and expansion of the leukemia cells in the blood and BM. Because LSCs are generally resistant to chemotherapy, it is thought that they are the underlying causes of therapeutic failure and disease recurrence in AML patients ${ }^{(17)}$.

The search for LSC-specific markers, or markers that show differential expression, has aroused strong enthusiasm in the fields of cancer therapy. Such markers can provide an excellent treatment option for targeting LSC, while preserving normal HSC ${ }^{(18)}$. These treatments should be substantially more acceptable for AML patients (19).

The stem cell-related expression of GPR56 was confirmed in a recent publication, but the lack of GPR56 was found to have no effect on the maintenance and function of HSC (20). It was shown that GPR56 is abundantly expressed by hematopoietic progenitor cells (HPCs) during definitive hematopoiesis in the embryo as well as the adult BM, but its levels are decreased substantially as HPCs differentiate (21). GPR56 was described to have a role in cell proliferation, survival, adhesion, and migration a variety of cell types ${ }^{(22)}$.

GPR56 was evaluated in several previous studies using PCR, while flow cytometry wasn't extensively evaluated. However, according to Pabst et al., who studied its flow cytometric expression in 45 AML patients, a highly significant positive correlation was found between the GPR56 gene expression and the GPR56\% of positive cells by flow cytometric immunophenotyping ( $\mathrm{r}=$ $0.9, P=0.001)^{(10)}$.

A significantly higher GPR56\% expression and MFI values were revealed in healthy control subjects than AML patients. Other studies showed comparable results to ours, though the lower expression of GPR56 gene expression in leukemic AML cells than normal myeloblasts wasn't interpreted ${ }^{(10,23-25)}$. For instance, Maiga et al. ${ }^{(23)}$ have evaluated the expression of GPCR in 148 AML samples using RNA-Seq; its expression was found to be downregulated in leukemic cells in comparison to that of normal cells (CD34+CD45RA-hematopoietic stem and progenitor cells derived from normal cord blood, as well as normal BM and PB cells). In the study of Pabst et al., GPR56 was found to be significantly expressed on the majority of normal CD34+ cells, implying that normal human stem and progenitor compartments are not further separated ${ }^{(10)}$.

Therefore, based on the finding of higher GPR56 levels in normal non-leukemic myeloblasts revealed in our study, we couldn't assign a cutoff point that would reliably define the positivity of GPR56 expression in our patients. We chose not to rely either on the traditional $20 \%$ cutoff point or any other alternative value to describe GPR56 expression, since there's no evidence in literature stating a cutoff point for GPR56 by flow cytometry. Therefore, samples were categorised as having low and high GPR56 expression based on expression levels that were lower or higher, respectively, than the overall median of GPR56 values in AML patients.

We compared levels of GPR56 expression in various prognostic categories. It was only the response to induction therapy that was affected by GPR56, with the latter having a significant negative impact on achieving CR. A cutoff value of $91.2 \%$ was assigned, which could fairly predict response to treatment at day 28 . To our knowledge, the predictive value of flow cytometric GPR56 expression in humans has not been previously investigated, although the effect of GPR56 gene expression on attaining CR in AML patients was concluded by Pabst $\boldsymbol{e t}$ al. ${ }^{(10)}$. On the other hand, GPR56 wasn't related to a prognostic cytogenetic aberration in our study. This didn't agree with the findings of Duncan $\boldsymbol{e t}$ al. (26) where higher levels of GPR56 gene expression was found among adverse and intermediate cytogenetic risk patient samples. In addition, Maiga et al. ${ }^{(23)}$ found that GPR56 gene is specifically overexpressed in FLT3-ITDmutated samples using RNA-Seq which impart a particularly poor prognosis. Also, Pabst et al. ${ }^{(10)}$ have correlated the missing or low expression of GPR56 gene to $\operatorname{inv}(16)$ and $t(8 ; 21)$, two cytogenetic abnormalities linked to a desirable prognosis. They also found high GPR56 expression in patients harboring poorly prognostic genetic abnormalities, including FLT3-ITD and RUNX1 and TP53 mutations. The controversy between the above findings and our results may be due to the limited number of our patients which might preclude a reliable representation of the different cytogenetic risk groups. 
The prognostic significance of the flow cytometric testing of GPR, which was the main target of this study, couldn't be fully established. No association was found between GPR56 levels and outcome of patients, patients' EFS, nor OS, as assessed in terms of maintenance of CR, relapse or death. However, in clear contradiction, the relationship between GPR56 gene expression and inferior disease consequences was concluded in several studies ${ }^{(18,27)}$. Still, these studies have documented the poor prognostic effect of GPR56 gene expression using PCR, so comparison with their results may not be fully reliable.

In conclusion, the association between GPR56 increased expression and failure to achieve CR presented in our study proposes a prognostic function of the flow cytometric GPR56 expression over AML patients' response to treatment at day 28 , which represents one important predictor of prognosis. Despite the lack of prognostic significance in terms of favorable and unfavorable patients' outcome or survival, and since response to treatment is an important predictor to outcome, GPR56 may represent an added indirect prognostic tool in the risk stratification of AML patients. Hence, patients with high GPR56 might be considered for stem cell transplantation or for novel investigative therapies. Further studies on a wider scale of AML patients, with extended periods of follow up, are recommended to validate the prognostic role of GPR56, assessed by flow cytometry.

It's of importance to state that the higher and brighter expression of GPR56 on myeloblasts of healthy non-AML subjects should be considered if the marker is used in flow cytometric follow up assessment of residual leukemia. This necessitates an extensive evaluation of GPR56 as a potential MRD marker as it might be a promising step in the challenging path of detection of residual leukemic cells in AML patients.

\section{REFERENCES}

1. Jemal A, Thomas A, Murray T, Thun M (2002): Cancer statistics, 2002. CA Cancer J Clin., 52(1):23-47.

2. Kantarjian H, Kadia T, DiNardo C et al. (2021): Acute myeloid leukemia: current progress and future directions. Blood Cancer J., 11: 41 .

3. Stone $\mathbf{R}$ (2009): Prognostic factors in AML in relation to (ab)normal karyotype. Best Pract Res Clin Haematol., 22(4):523-8.

4. Herrmann H, Blatt K, Shi J et al. (2012): Small-molecule inhibition of BRD4 as a new potent approach to eliminate leukemic stem- and progenitor cells in acute myeloid leukemia (AML). Oncotarget, 3(12):1588-1599.

5. Eppert K, Takenaka K, Lechman E et al. (2011): Stem cell gene expression programs influence clinical outcome in human leukemia. Nat Med., 17(9):1086-1093.

6. Dick J (2008): Stem cell concepts renew cancer research. Blood, 112(13):4793-4807.

7. Jin L, Hope K, Zhai Q et al. (2006): Targeting of CD44 eradicates human acute myeloid leukemic stem cells. Nat Med., 12:1167-1174.

8. Iguchi T, Sakata K, Yoshizaki K et al. (2008): Orphan G protein coupled receptor GPR56 regulates neural progenitor cell migration via a $\mathrm{G}$ alpha $12 / 13$ and Rho pathway. J Biol Chem., 283:14469-14478.
9. Ke N, Sundaram R, Liu G et al. (2007): Orphan G proteincoupled receptor GPR56 plays a role in cell transformation and tumorigenesis involving the cell adhesion pathway. Mol Cancer Ther., 6:1840-1850.

10. Pabst C, Bergeron A, Lavallée V et al. (2016): GPR56 identifies primary human acute myeloid leukemia cells with high repopulating potential in vivo. Blood, 127(16):2018-2027.

11. Arber D, Brunning R, Le beau M et al. (2017): Acute Myeloid Leukemia and related precursor neoplasms. In: WHO Classification of Tumours of Haematopoietic and Lymphoid Tissue. Swerdlow SH, Campo E, Harris NL et al. (eds). Fifth edition. WHO press, Switzerland. Chapter 8; 141-142.

12. National Comprehensive Cancer Network Guidelines in Oncology (2013): Acute Myeloid Leuekmia. www.nccn.org/professionals/physician gls/PDF/aml.pdf.

13. Matutes E, Morillo R, Cotovsky D (2005): Immunophenotypic analysis of acute myeloid leukemia. In: Practical Hematology. 10th ed. Lewis S. Bain B. and Bates I. (eds). Harcourt Publishers Limited, England p. 335.

14. Grimwade D, Hills R, Moorman A et al. (2010): Refinement of cytogenetic classification in acute myeloid leukemia: determination of prognostic significance of rare recurring chromosomal abnormalities among 5876 younger adult patients treated in the United Kingdom Medical Research Council trials. Blood, 116(3):354-365.

15. Döhner H, Estey D, Grimwade D et al. (2017): Diagnosis and management of AML in adults: 2017 ELN recommendations from an international expert panel. Blood, 129:424-447.

16. Padilha S, Souza E dos S, Matos M, Domino N (2015): Acute myeloid leukemia: survival analysis of patients at a university hospital of Paraná. Revista Brasileira de Hematologia e Hemoterapia, 37(1):21-27.

17. Zhou J, Cheng W (2014): Identification and targeting leukemia stem cells: The path to the cure for acute myeloid leukemia. World J Stem Cells., 6(4):473-84.

18. Pabst C, Krosl J, Fares I et al. (2014): Identification of small molecules that support human leukemia stem cell activity ex vivo. Nat Methods., 11(4):436-442.

19. Witte K, Ahlers J, Schäfer I et al. (2011): High proportion of leukemic stem cells at diagnosis is correlated with unfavorable prognosis in childhood acute myeloid leukemia. Pediatr Hematol Oncol., 28: 91-99.

20. Rao T, Marks-Bluth J, Sullivan J et al. (2015): High-level Gpr56 expression is dispensable for the maintenance and function of hematopoietic stem and progenitor cells in mice. Stem Cell Res., 14:307-322.

21. Gazit R, Garrison B, Rao T et al. (2013): Transcriptome analysis identifies regulators of hematopoietic stem and progenitor cells. Stem Cell Rev., 1:266-280.

22. Krishnan A, Nijmeijer S, de Graaf C et al. (2016): Classification, nomenclature, and structural aspects of adhesion GPCRs. In: Langenhan T., Schöneberg T. (eds) Adhesion G Protein-coupled receptors. Handbook of Experimental Pharmacology, vol 234. Springer, Cham.

23. Maiga A, Lemieux S, Pabst $C$ et al. (2016): Transcriptome analysis of $\mathrm{G}$ protein-coupled receptors in distinct genetic subgroups of acute myeloid leukemia: identification of potential disease-specific targets. Blood Cancer J., 6(6):e431.

24. Seita J, Sahoo D, Rossi D et al. (2012): Gene expression commons: an open platform for absolute gene expression profiling. PLos One, 7:e40321.

25. Daria D, Kirsten N, Muranyi A et al. (2016): GPR56 contributes to the development of acute myeloid leukemia in mice. Leukemia, 30(8):1734-41.

26. Duncan H, Hermans K, Chisling S et al. (2016): G proteincoupled receptor 56 as a potential regulator of normal and leukemic stem cells. Blood, 126(23):4267-4267.

Luo R, Jeong S, Jin Z et al. (2011): G protein-coupled receptor 56 and collagen III, a receptor-ligand pair, regulates cortical development and lamination. Proc Natl Acad Sci USA., 108:12925-12930. 